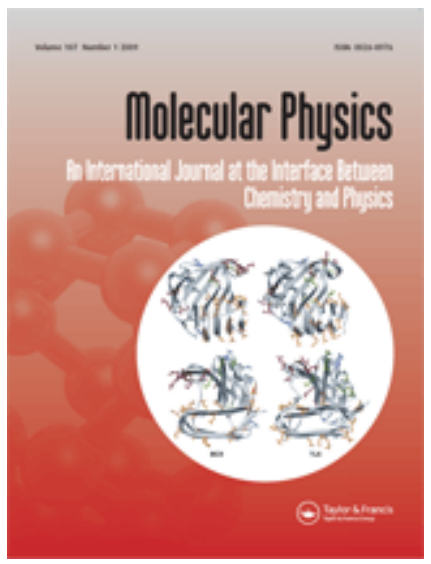

\title{
First-Principle Computations of Rotational-Vibrational Transition Probabilities
}

\begin{tabular}{|r|l|}
\hline Journal: & Molecular Physics \\
\hline Manuscript ID: & TMPH-2008-0081 \\
\hline Manuscript Type: & Full Paper \\
\hline Author: & 12-Mar-2008 \\
\hline Kemplete List of Authors: & $\begin{array}{l}\text { Linguerri, Roberto; Universite Paris-Est, Equipe de Chimie } \\
\text { Theorique }\end{array}$ \\
\hline \multicolumn{2}{|c|}{$\begin{array}{l}\text { radiative transition probabilities, ions, spectroscopy, molecular } \\
\text { physics }\end{array}$} \\
\hline $\begin{array}{l}\text { Note: The following files were submitted by the author for peer review, but cannot be converted } \\
\text { to PDF. You must view these files (e.g. movies) online. }\end{array}$ \\
\hline \begin{tabular}{l} 
manuscript.tex \\
\hline
\end{tabular}
\end{tabular}

\section{scholarONE" \\ Manuscript Central}




\title{
First-Principle Computations of Rotational-Vibrational Transition Probabilities
}

\author{
Pavel Rosmus* and Roberto Linguerri \\ Laboratoire de Chimie Théorique, \\ Université de Marne la Vallée, \\ F 77454 - Champs sur Marne, France \\ Najia Komiha \\ Laboratoire de Chimie Théorique, \\ Université Mohamed V-Agdal,Rabat, \\ Morocco
}

(Dated: March 12, 2008)

\begin{abstract}
The theoretical calculations of dipole moments provide reliable compact information required for the evaluation of radiative transition probabilities in the electronic states of small molecular ions. The comparison of the computed line intensities and dipole derivatives with available experimental data in neutral molecules demonstrates that they can be calculated $a b$ initio with a precision of a few percent, hence with an accuracy sufficient for most of the experimental applications.
\end{abstract}

PACS numbers:

\section{INTRODUCTION}

The high quality first-principle quantum mechanical calculations of the absolute radiative transition probabilities in the electronic states of molecular ions yield more accurate results than experiments. Similar to neutral molecules such data are needed in many fields of research, for instance, for assignments of high resolution spectra, spectral line broadening, measurements of ion abundances, radiative decay rates of excited rotational-vibrational states, in atmospheric chemistry, astrophysics, or in studies of ion-molecule reactions. In order to show how accurately the radiative transition probabilities can be calculated by a purely theoretical approach for small molecular ions, we first discuss the results for neutral triatomics $\mathrm{O}_{3}[1], \mathrm{H}_{2} \mathrm{~S}[2]$, and $\mathrm{CO}_{2}[3]$ because of the numerous precise spectral data[4-6] available for comparison. In the first-principle approach the potential energy and electric dipole moments surfaces are computed from highly correlated electronic wavefunctions[7]. This compact information is then used to solve the nuclear motion problem variationally, using exact kinetic energy operator in internal coordinates[8-10], yielding rotational-vibrational energies and transition dipole matrix elements. Such a variational approach is essential for accurate results.

\section{OZONE}

In the analysis of the infrared spectra of the electronic ground state of ozone[11] usually Hamiltonian matrices are set up for polyads of Darling-Dennison and Coriolis

*Electronic address: rosmus@univ-mlv.fr resonances, and diagonalized. Using the mixing coefficients, the necessary spectroscopic data are evaluated for the line assignments and the transition moment parameters. From the least-squares fitting of the transition moment parameters to the measured subset of line intensities one can calculate all line intensities for a given vibrational band. The experimental data were used to set up an effective electric dipole moment function[12] expanded as a linear combination in the normal modes and the corresponding dipole derivatives. At most, one derivative is evaluated from the transition moment parameters of one vibrational band. For instance, the second derivatives were obtained from the first overtones or corresponding combination levels.

We have calculated more than $4.8 \times 10^{6}$ electric dipole transition matrix elements for the absolute rotationalvibrational line intensities of all bound levels up to about $8500 \mathrm{~cm}^{-1}$ and $\mathrm{J}=64$. All anharmonic and Coriolis resonances were implicitly taken into account in the variational calculations with the nuclear motion Hamiltonian. In Figure 1 the calculated pattern of rotational levels up to $\mathrm{J}=64$ for the vibrational ground state is shown. Since the value of dissociation energy of ozone is about $8500 \mathrm{~cm}^{-1}$, all rotational-vibrational states with lower $\mathrm{J}$ will play a role in the energy redistribution during the formation of ozone in vibrational ground state by threebody collisions of the $\mathrm{O}_{2}-\mathrm{O}$ complex with other atoms and molecules. In the calculations of the line intensities at $273 \mathrm{~K}$, we used the value of the partition function from the HITRAN database. In all calculations of absorption spectra a Boltzmann distribution at $273 \mathrm{~K}$ was assumed, although the dipole matrix elements could serve in calculations of emission or absorption spectra for any population of the initial level.

In order to compare the calculated dipole functions with previous theoretical and empirical functions, we have transformed our dipole function to the Eckart frame and used our harmonic wavefunctions to calculate the deriva- 
FIG. 1: Pattern of variationally calculated rotational $\mathrm{K}_{a} \mathrm{~K}_{c}$ levels up to $\mathrm{J}=64$ in the vibrational ground state of ${ }^{16} \mathrm{O}_{3}$. Starting from $\mathrm{J}=46$ highest levels are not plotted

TABLE I: First and second derivatives with respect to dimensionless normal coordinates of the electric dipole moment function of ozone in Eckart frame (in $10^{-2}$ debye)[1].

\begin{tabular}{lcccc}
\hline \hline & MRCI & MRCI+Q $^{a}$ & MRCI+Q [13] & Exp. $[12]$ \\
\hline$\mu_{1}^{x}$ & -1.839 & -2.097 & -1.897 & -2.185 \\
$\mu_{2}^{x}$ & -7.178 & -6.812 & -7.456 & -6.529 \\
$\mu_{11}^{x}$ & -0.409 & -0.344 & -0.476 & -0.366 \\
$\mu_{12}^{x}$ & 0.828 & 0.786 & 0.777 & 0.894 \\
$\mu_{22}^{x}$ & -0.145 & -0.154 & -0.097 & -0.122 \\
$\mu_{33}^{x}$ & 0.756 & 1.149 & 0.662 & 0.769 \\
$\mu_{3}^{z}$ & -26.92 & -25.40 & -28.85 & -26.62 \\
$\mu_{13}^{z}$ & 4.98 & 4.85 & 4.50 & 4.81 \\
$\mu_{23}^{z}$ & -0.258 & -0.559 & 0.381 & 0.154 \\
\hline \hline
\end{tabular}

${ }^{a}$ The data in the third column have been recalculated from the dipole moment function of [13].

tives with respect to dimensionless normal coordinates. These values are given in Table I. The derivatives from experiment are in good overall agreement with our theoretical values as far as the signs and absolute values are concerned.

For atmospheric applications it is necessary to know the intensities with an accuracy better than 1 to 3 percent. In Figures 2 and 3 the first-principle rotationally resolved absorption spectra of ozone are compared with the spectra from the HITRAN database. Both sets of spectral intensities are found to be in excellent agreement. For purely rotational transitions the differences are about 0.3 percent. For the transitions to (010), (001), (110), (011) the agreement is within 1 to 3 percent. Surprisingly, also, the extremely weak transitions to the (020) state ( the line intensities are ten thousands times weaker than those of the fundamental antisymmetric stretching transition)
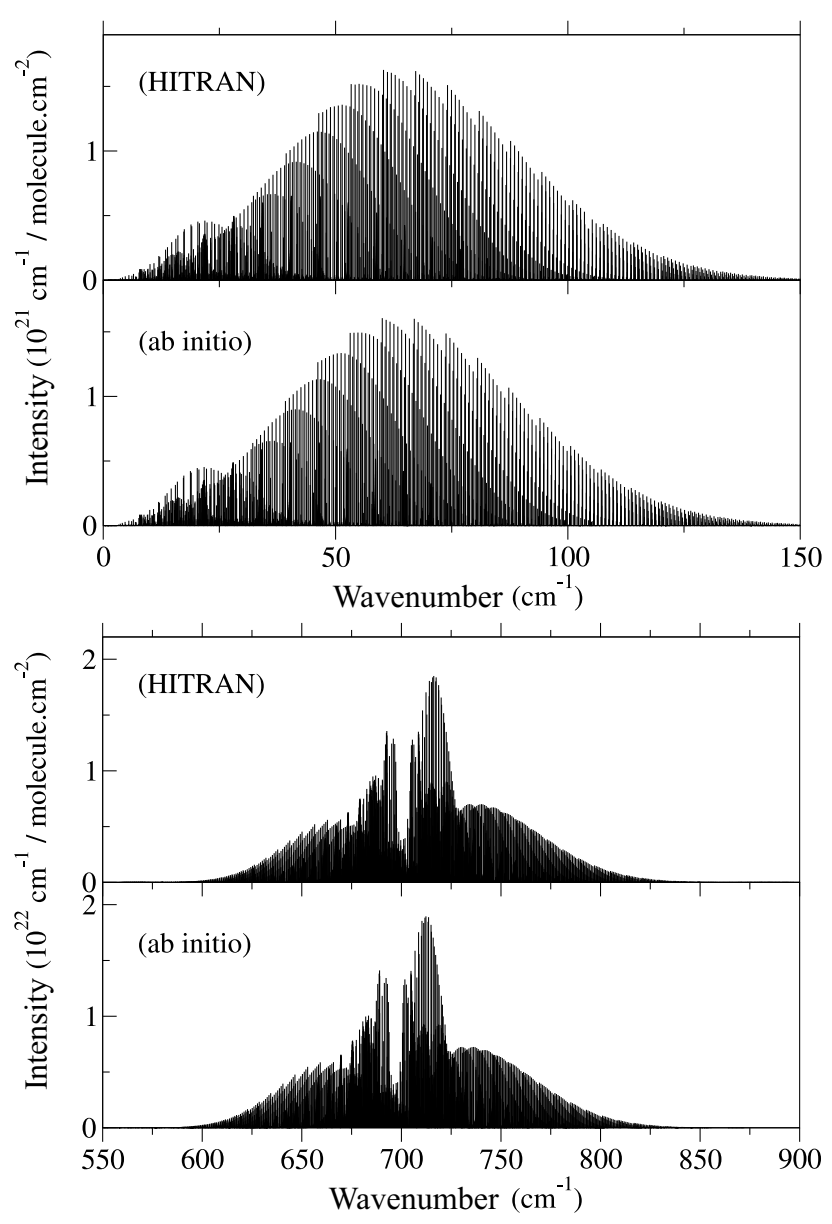

FIG. 2: Purely rotational absorption spectrum at $273 \mathrm{~K}$ (upper part) and bending fundamental (lower part).

agree very well with the database values. Very pleasing is the agreement between theory and experiment for the (001) band also on the large scale for transitions lying within about $10 \mathrm{~cm}^{-1}$ in Figure 4 . The ozone spectrum exhibits congested regions where several lines lie within 1 $\mathrm{cm}^{-1}$ of each other. The first-principle variational calculations for a molecule with three heavy atoms are unable to yield the proper ordering of such states, although the line intensities are still correctly calculated and useful for assignments.

\section{HYDROGEN SULFIDE}

The experimental infrared spectra of $\mathrm{H}_{2} \mathrm{~S}$ molecule present interesting anomalies in the rovibrational intensity distributions[2]. They concern an unusual behaviour of band intensities versus a number of vibrational quanta $\Delta v=\Delta v_{1}+\Delta v_{2}+\Delta v_{3}$ : observed intensities of $\mathrm{H}_{2} \mathrm{~S}$ fundamental bands $(\Delta v=1)$ are much weaker than those of combination bands with $\Delta v=2$. For example, $\mathrm{P}_{1}$ transitions of $\nu_{1}$ and $\nu_{3}$ fundamental bands are by one or two orders of magnitude weaker than those of $\nu_{1}+\nu_{2}, \nu_{2}+\nu_{3}$, 

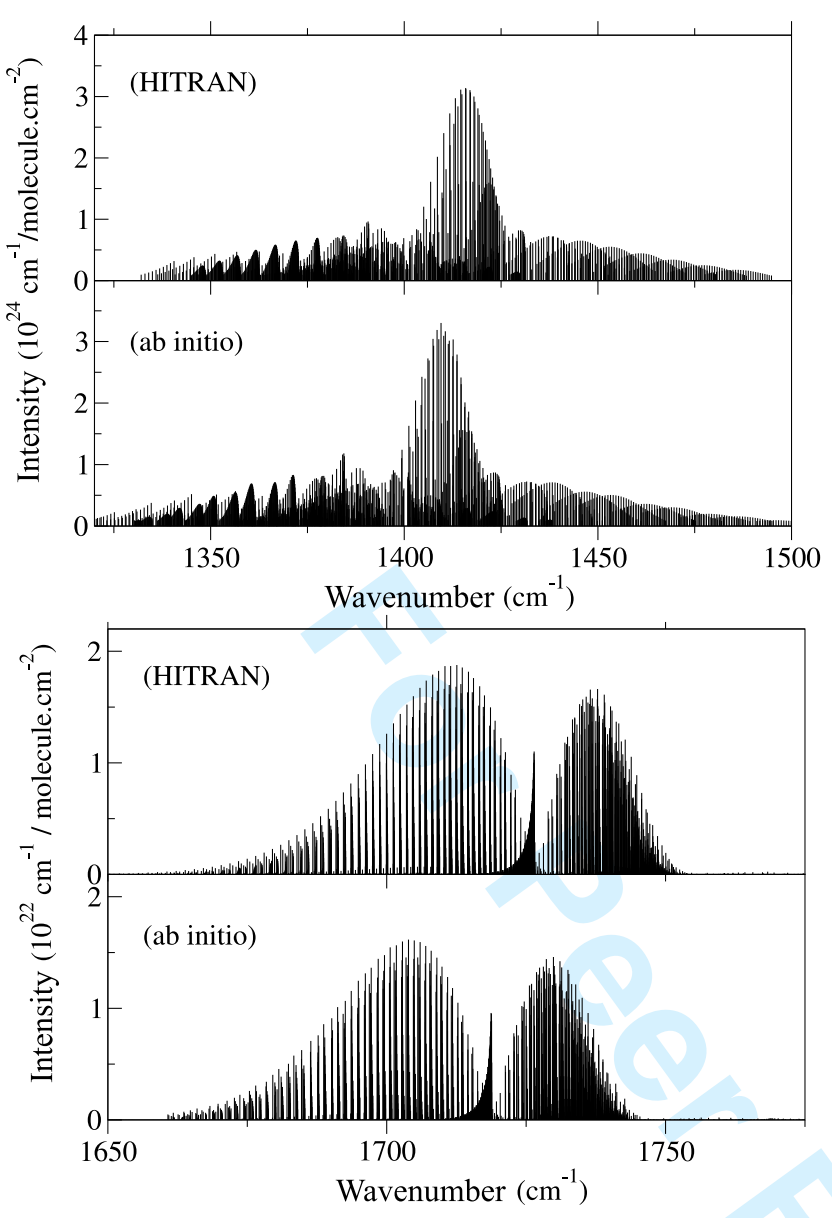

FIG. 3: Absorption spectra at $273 \mathrm{~K}$ for the combination (020) band (upper part) and (011) band (lower part).

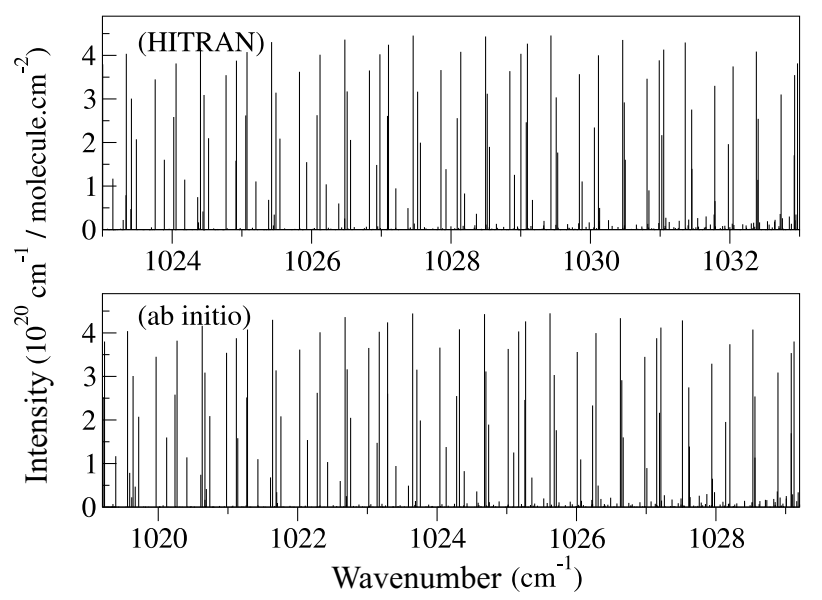

FIG. 4: Part of the absorption spectrum for the antisymmetric stretching fundamental. Upper spectrum has been taken from the HITRAN database and lower is from the firstprinciples computations.

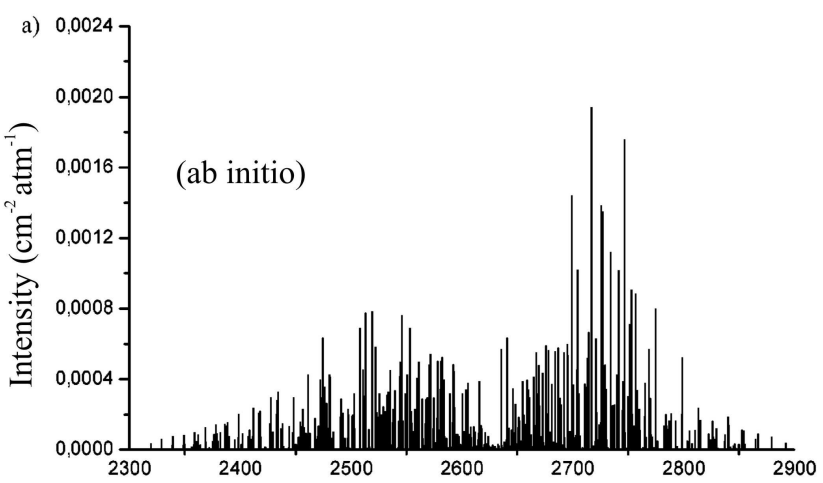

b)

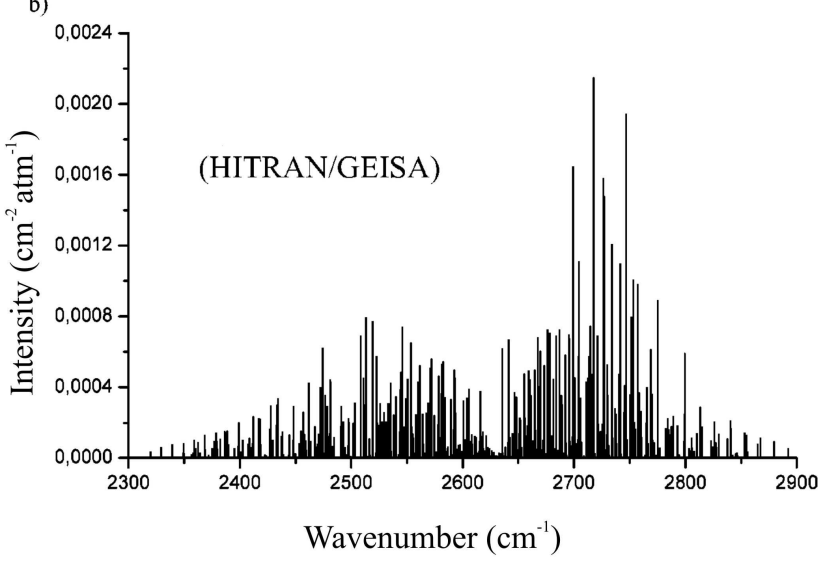

FIG. 5: Absorption spectrum at $273 \mathrm{~K}$ for the $\nu_{3}$ band; $J_{\text {upper }} \leq 10$, Jlower $\leq 11$.

or $\nu_{1}+\nu_{3}$ combination bands unlike the majority of bent triatomic molecules. The fundamental band $\Delta v_{3}=1$ is particularly weak, The experimentally measured fundamental band $\Delta v_{3}=1$ is weaker than the corresponding transitions of $\Delta v=2$ bands, many $\Delta v=3$ bands and even of some $\Delta v=4$ bands. Fundamental bands exhibit also rotational anomalies in intensity distributions. The P-branches of the $\nu_{1}$ and $\nu_{2}$ bands are much less intense than the R-branches and in the $\nu_{3}$ band some $\Delta \mathrm{K}_{a}=2$ transitions are more intense than corresponding $\Delta \mathrm{K}_{a}=0$ transitions. The components of the dipole moment function are very flat close to the equilibrium geometry, therefore contributions to transition dipole matrix elements of fundamental bands $\nu_{1}, \nu_{3}$ from second and higherorder derivatives with respect to normal or displacement coordinates become comparable and even more important than the first derivatives. All anomalies are due to the unusual form of this function. The coupled cluster method with perturbative triples has been employed with a large basis set augmented by diffuse functions and the components of the dipole moment have been evaluated as the energy derivatives with respect to an applied electric field. In Figure 5 we compare the rotationally resolved absorption spectrum of the very weak $\nu_{3}$ band calculated $a b$ initio with the spectrum available in the data bases. The overall agreement is considered as very 
good. Already an error of about $10^{-2}$ debye $/ \AA$ produces a very large deviations in the calculated line intensities. Other examples are given in Ref.[2]. The dipole moment function for the electronic ground state of hydrogen sulfide is computationally extremely challenging task due to the accuracy required for the evaluations of radiative transition probabilities particularly for higher frequency regions.

\section{DIPOLE MOMENTS OF SMALL IONS}

The electric dipole moments in the vibrational ground states are of importance, for instance, in microwave spectroscopy or radioastronomy. They can be determined experimentally only in indirect way. The $\mathrm{CO}^{+}$ dipole moment[14] was deduced from polarizabilities and precise mass comparison of $\mathrm{CO}^{+}$to $\mathrm{N}_{2}^{+}$. The cyclotron frequency shifts between $\mathrm{N}=0$, and $\mathrm{N}=1$, $\mathrm{M}_{N}=0, \pm 1$ states yield a value of $\mu_{0}=1.025(15)$ ea $\mathrm{a}_{0}$, which compares well with our recent MRCI theoretical value of $1.035(8) \mathrm{ea}_{0}[15]$. Prior to the measurements of polarizability shifts of $\mathrm{CO}^{+}$, experimental determination of dipole moments in diatomic molecular ions have been based on isotopic substitution in the rotational Zeeman effect, or else extensive analysis of pure-rotation na rotation-vibration spectra of isotopic variants. The example of $\mathrm{ArH}^{+}$, which received most attention, shows the difficulties with previous experimental methods. The experimentally determined dipole moments of 1.4(0.4) debye[16] and 3.0(0.6) debye[17] may be compared with our recent MRCI result of 2.177 debye and the experimental determination from detailed spectral analysis $2.12(0.55)$ debye[18] ( 1 ea $0=2.54158$ debye $)$.

The most complete and accurate theoretical study of emission rates for all rotation-vibration transitions has been reported for $\mathrm{HeH}^{+}[19]$. This ion is thought to be the first molecular species to appear in the Universe. Consequently, stars formed from primordial material should contain some $\mathrm{HeH}^{+}$. These theoretical predictions should help to determine the best conditions to search for and detect the ion. It should also facilitate its detection in other astrophysical environments.

Recently theoretical electric dipole moment functions of the first - row diatomic hydride ions have been reported. The dipole moments in the vibrational ground states are given in Table II. The $\mathrm{LiH}^{+}$ion is thought to be constituent of the primordial matter like $\mathrm{HeH}^{+}$. The calculated dipole moment function is, however, very flat near the equilibrium distance and the dipole moment $\mu_{0}$ is not very large. The protonating mode in $\mathrm{HeH}^{+}$has much stronger vibrational transitions and it seems to be hardly probable to detect $\mathrm{LiH}^{+}$in infrared spectral region of the electromagnetic radiation from outer space. The parts of the dipole moment functions near the equilibrium geometries of the ions in Figure 6 reflect also the first ionization energies of the heavy atoms relative to hydrogen. The dipole moments of ions are

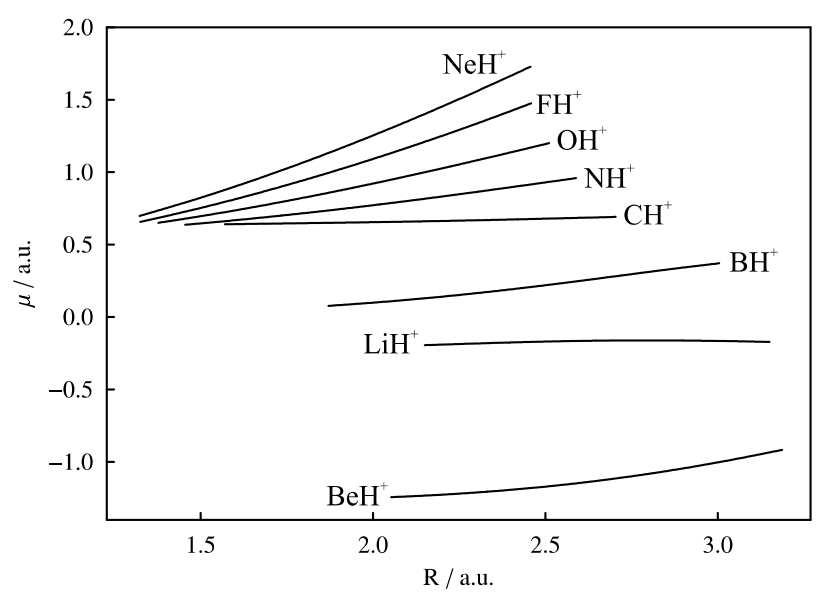

FIG. 6: Dipole moment as a function of internuclear separation: $\mathrm{LiH}^{+}$to $\mathrm{NeH}^{+}$.

calculated for each isotope variant with origin at the center of mass. Asymptotically they are zero for $r=0 \AA$ and go to infinity for large internuclear distances. They can, however, possess a maxima or minima which will influence the infrared spectra in high frequency regions.

The dipole moments of the hydride ions were needed in calculations of orientation polarizabilities for very precise atomic mass measurements[15]. The bottleneck of this approach is the accuracy of the theoretical dipole moments which are expected to be \pm 0.008 ea $_{0}$

Several dipole moment functions of molecular ions have been used to calculate radiative relaxation of vibrational states. Perhaps the best known example is $\mathrm{NO}^{+}$for which the decay rates for five vibrational levels have been measured by monitoring the change in vibrational populations through internal energy dependent fast reactions. The latest experimental[20] and theoretical[21] radiative lifetimes for $v=1$ to 5 lying in the range of 90 to $20 \mathrm{~ms}$ were found to be in good overall agreement. To our knowledge no other experimental information about the shapes of larger parts of the dipole moment funcitions in molecular ions have been reported so far. Though recent experiments[22] proved the existence of six equivalent $\mathrm{C}_{2 v}$ minima in the pseudorotating $\mathrm{CH}_{4}^{+}$ ion no transition probabilities are known for this important species. In a series of $\operatorname{RCCSD}(\mathrm{T})$ computations with cc- VxZ AO basis sets we have reoptimised the equilibrium geometry of the lowest $\mathrm{C}_{2 v}$ minimum of $\mathrm{CH}_{4}^{+}$. Our best $\mathrm{r}_{e}$ geometry is for the two $\mathrm{CH}_{2}$ angles $62.76^{\circ}$ and $27.55^{\circ}$ and the two $\mathrm{CH}$ distances $1.0796 \AA$ and $1.1825 \AA$ (cf. ref. for the definition of the geometry parameters). This $\mathrm{CH}_{4}^{+}$structure has an unexpectedly large dipole moment of $-0.6600 \mathrm{ea}_{0}$, the $\mathrm{CD}_{2} \mathrm{H}_{2}^{+}-0.7788$ $\mathrm{ea}_{0}$. Regardless of complicated intramolecular dynamics playing a role in the radiative transition probabilities the large dipole advocates the possibility to detect the ion in the outer space. 
TABLE II: Calculated MRCI electric dipole moments $\mu_{0}$, and internuclear separations $R_{0}$, for the lowest vibrational state of $\mathrm{CO}^{+}$and the hydride cations $\mathrm{LiH}^{+}$to $\mathrm{NeH}^{+}$. A positive sign for the dipole implies it is directed towards the lighter nucleus. $\mu_{0}$ and $R_{0}$ are given in atomic units $\left(e a_{0}, a_{0}\right), B_{0}$ is given in $\mathrm{cm}^{-1}$ (see Ref. [15] for details).

\begin{tabular}{lcccc}
\hline \hline $\begin{array}{l}\text { Diatomic } \\
\text { ion }\end{array}$ & State & $\mu_{0}$ & $R_{0}$ & $\begin{array}{c}R_{0} \\
\text { Exp. }\end{array}$ \\
\hline${ }^{12} \mathrm{C}^{16} \mathrm{O}^{+}$ & & 1.0352 & 2.1160 & 2.1125 \\
Exp.
\end{tabular}

\section{INFRARED EMISSION IN THE $\mathrm{X}^{2} \Pi_{g}$ STATE $\mathrm{CO}_{2}^{+}$}

Spacecraft observations of $\mathrm{CO}_{2}^{+}$in Mars' atmosphere have been made for more than 35 years (see Ref. [3] for a review). The densities of the principal ions $\left(\mathrm{O}_{2}^{+}\right.$, $\left.\mathrm{CO}_{2}^{+}, \mathrm{O}^{+}\right)$in Mars' atmosphere were measured by the Retarding Potential Analyzers on the Viking 1 and 2 landers on their descent. The production rate in planetary atmospheres of the $\mathrm{CO}_{2}^{+}$ion has been determined by using ultraviolet emission bands.

The Thermal Emission Spectrometer on Mars Global Surveyor has observed numerous limb spectra of Mars' atmosphere from near the surface to above $125 \mathrm{~km}$. The resulting averaged IR emission spectrum covers an energy range from $150-1650 \mathrm{~cm}^{-1}$ and shows the strong $\mathrm{X}^{1} \Sigma_{g}^{+} \mathrm{CO}_{2}(001) \rightarrow(100)-(020)$ Fermi resonance pair near 960 and $1063 \mathrm{~cm}^{-1}$ and the $\mathrm{X}^{1} \Sigma_{g}^{+} \mathrm{CO}_{2}$ fundamental bending transition at $667 \mathrm{~cm}^{-1}$. The IR emission spectrum has also features in the energy range 400-500 $\mathrm{cm}^{-1}$.

Using $a b$ initio calculated potential energy and electric dipole and transition moment functions, absolute radiative transition probabilities between the rovibronic Renner-Teller states of the electronic ground state $\mathrm{X}^{2} \Pi_{g}$ of $\mathrm{CO}_{2}^{+}$have been evaluated. The rovibronic eigenstates were obtained up to $6500 \mathrm{~cm}^{-1}$ and $J \leq 13 / 2$ from variational calculations including the Renner-Teller and spin-orbit angular momentum couplings. The absolute intensities for the rotational-vibrational transitions in the neutral $\mathrm{CO}_{2}$ electronic ground state[3], calculated by a similar approach, are found to be in excellent agreement with experimental data. For $\mathrm{CO}_{2}^{+}$the presented intensities are expected to have similar accuracy. The calculations show that overtone intensities of the antisymmetric stretching mode of $\mathrm{CO}_{2}^{+}$are of similar magnitude as the fundamental transitions, in contrast with $\mathrm{CO}_{2}$.

The derivatives of the dipole moment functions with respect to the dimensionless normal coordinates at the equilibrium geometry of the electronic ground states

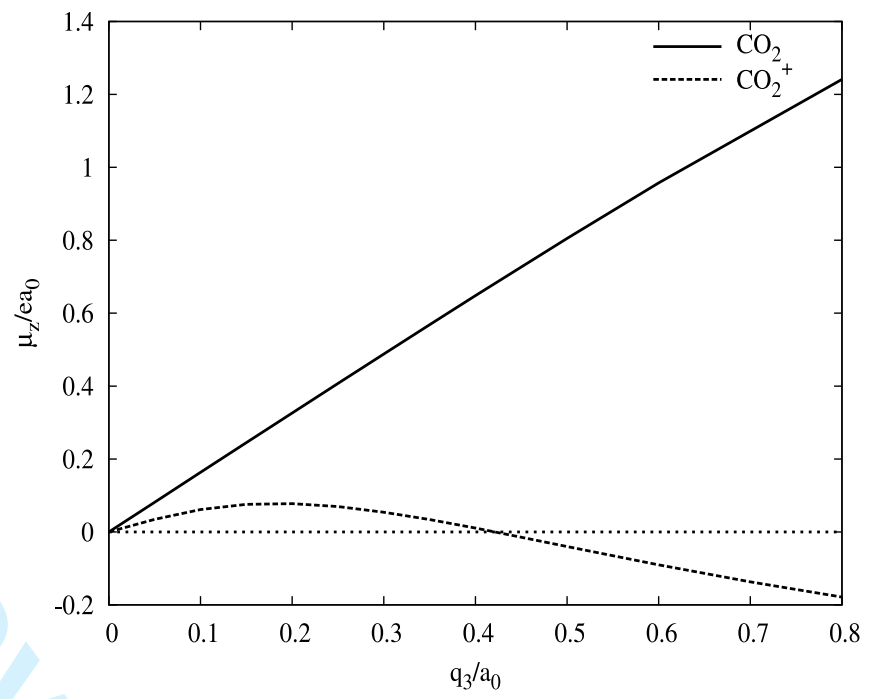

FIG. 7: $\mu_{z}$ along the antisymmetric coordinate $q_{3}$ using MCSCF/cc-pVQZ method. $q_{3}=\left(r_{2}-r_{1}\right) / \sqrt{2}$ and $q_{1}=$ $\left(r_{2}+r_{1}\right) / \sqrt{2}$ is fixed at the equilibrium value.

of $\mathrm{CO}_{2}$ and $\mathrm{CO}_{2}^{+}$are given in Tables III and IV, respectively. The $a b$ initio values for $\mathrm{CO}_{2}$ are compared with previous data extracted from experimental measurements using direct numerical diagonalization (DND) (cf. Ref. [3]). Both sets are in excellent agreement. For $\mathrm{CO}_{2}$, the second and third derivatives are always smaller than the first ones. The $\mu_{3}^{z}$ derivative is more than twice as large as $\mu_{2}^{x}$ reflecting larger radiative probabilities for transitions involving the antisymmetric stretching mode compared to the bending mode.

In Table IV the same derivatives are given for both electronic components of $\mathrm{CO}_{2}^{+}$. The two sets of derivatives are similar. The $\mu_{y}$ derivatives are always much smaller than other derivatives. The $\mu_{x}$ derivatives of $\mathrm{CO}_{2}^{+}$are similar to the $\mu_{x}$ derivatives of $\mathrm{CO}_{2}$. The comparison of the derivatives for $\mu_{z}$ between $\mathrm{CO}_{2}$ and $\mathrm{CO}_{2}^{+}$reveals unexpectedly large third derivatives with respect to the dimensionless antisymmetric stretching 
TABLE IV: MRCI derivatives of the dipole moment functions $\left(10^{-2}\right.$ debye $)$ for the $\mathrm{X}^{2} \Pi_{g}$ of $\mathrm{CO}_{2}^{+}$with respect to the dimensionless normal coordinates at the equilibrium geometry $\left(R_{C O}=2.2207 \mathrm{a}_{0}\right)[3]$. normal coordinates at the equilibrium geometry $\left(R_{C O}=2.1970 \mathrm{a}_{0}\right)[3]$.

\begin{tabular}{|c|c|c|c|c|c|}
\hline \multicolumn{3}{|c|}{$\mu_{x}$} & & \multicolumn{2}{|c|}{$\mu_{z}$} \\
\hline & $\operatorname{CCSD}(\mathrm{T})$ & Exp.(DND) & & $\operatorname{CCSD}(\mathrm{T})$ & Exp.(DND) \\
\hline$\mu_{2}$ & 18.48 & 18.4 & $\mu_{3}$ & -45.65 & -46.1 \\
\hline$\mu_{12}$ & -0.78 & -0.783 & $\mu_{13}$ & 1.86 & 1.82 \\
\hline$\mu_{112}$ & 0.004 & 0.00591 & $\mu_{113}$ & 0.03 & 0.00518 \\
\hline$\mu_{233}$ & 0.16 & & $\mu_{223}$ & 0.73 & 0.668 \\
\hline$\mu_{222}$ & -0.93 & -0.652 & $\mu_{333}$ & 0.17 & 0.151 \\
\hline
\end{tabular}

\begin{tabular}{|c|c|c|c|c|c|}
\hline \multicolumn{4}{|c|}{$\mu_{x}$} & \multicolumn{2}{|c|}{$\mu_{z}$} \\
\hline & $A^{\prime}$ & $A^{\prime \prime}$ & & $A^{\prime}$ & $A^{\prime \prime}$ \\
\hline$\mu_{2}$ & 25.25 & 21.71 & $\mu_{3}$ & -24.40 & -24.40 \\
\hline$\mu_{12}$ & -1.20 & -0.86 & $\mu_{13}$ & -1.35 & -1.35 \\
\hline$\mu_{112}$ & -0.02 & -0.24 & $\mu_{113}$ & -0.12 & -0.12 \\
\hline$\mu_{233}$ & 1.48 & 1.62 & $\mu_{223}$ & 0.91 & 0.77 \\
\hline$\mu_{222}$ & -1.48 & -1.06 & $\mu_{333}$ & 28.42 & 28.42 \\
\hline & $A^{\prime a}$ & $A^{\prime \prime b}$ & & & \\
\hline$\mu_{2}$ & -0.64 & -0.58 & & & \\
\hline$\mu_{12}$ & 0.16 & 0.14 & & & \\
\hline$\mu_{222}$ & 0.07 & 0.05 & & & \\
\hline$\mu_{112}$ & -0.04 & -0.04 & & & \\
\hline$\mu_{233}$ & -0.32 & -0.29 & & & \\
\hline
\end{tabular}

${ }^{a}$ derivatives with respect to the normal coordinates of $A^{\prime}$ component.

${ }^{b}$ derivatives with respect to the normal coordinates of $A^{\prime \prime}$ component.

coordinate in $\mathrm{CO}_{2}^{+}$. In Figure $7, \mu_{z}$ is plotted against $q_{3}=\left(r_{2}-r_{1}\right) / \sqrt{2}$ for $\mathrm{CO}_{2}$ and $\mathrm{CO}_{2}^{+}$. The curves show that $\mu_{z}$ is nearly linear with a large slope for $\mathrm{CO}_{2}$ whereas $\mu_{z}$ has a shallow maximum close to the linear structure. Similar to $\mathrm{H}_{2} \mathrm{~S}$ rovibronic intensity anomalies are to be expected for the transition probabilities including the antisymmetric stretching mode.

As an illustration of the results, a compact representation in form of the radiative lifetimes of the first rotational-vibrational and rovibronic states of $\mathrm{CO}_{2}$ and $\mathrm{CO}_{2}^{+}$for $J=|P|$ are shown in Figure 8. The $v_{2}$ transitions are connected. The lifetimes decrease with the values of $v_{2}$ and $v_{1}$ (if $v_{1}>0$ ) in the neutral and the ionic species. In $\mathrm{CO}_{2}$ the lifetimes for levels involving the antisymmetric stretching mode are smaller than $3 \mathrm{~ms}$ whereas they are about $70 \mathrm{~ms}$ in $\mathrm{CO}_{2}^{+}$. In Figure 8 (lower part), the outliers are due to Fermi coupling. Particularly, the radiative lifetime of the level $\Pi_{g 3 / 2}(1,0,0)$ is lower than the one of the level $\Pi_{g 1 / 2}(1,0,0)$, although its energetic position is lower.

The Mars spectra from the middle atmosphere through about $127 \mathrm{~km}$ show a strong broad structured peak extending from $\sim 420-\sim 520 \mathrm{~cm}^{-1}$. The feature is optically thin so limb tangent observations include emission from many altitudes, including the $\mathrm{CO}_{2}^{+}$peak at $\sim 130 \mathrm{~km}$. For the comparison of the calculations to these observations, the spontaneous emission rates of $\mathrm{CO}_{2}^{+}$for equal initial population of the excited levels and for the values of $J 1 / 2 \leq J \leq 5 / 2$, with the maximum energy at 2000 $\mathrm{cm}^{-1}$ were used together with the energy positions to create a synthetic theoretical spectrum (cf. Ref.[3] for details). The envelope considering all dipole allowed transitions adapted to the resolution of the experiment is plotted in figure 9 .

Both the spontaneous rates and the synthetic spectrum reproduce features between 465 and $509 \mathrm{~cm}^{-1}$. The transitions are between levels involving the bending quantum number $v_{2}$, with $\Delta v_{2}= \pm 1$ and the complicated structures are the result of Fermi, spin-orbit and Renner-Teller couplings. $\mathrm{CO}_{2}^{+}$has also significant emission in the $1000 \mathrm{~cm}^{-1}$ region where neutral $\mathrm{CO}_{2}$ hot bands are present. Other parts of the broad feature are less well reproduced, indicating nonthermal population of the $\mathrm{CO}_{2}^{+}$energy levels and/or additional emission sources. In comparing the peak positions of the synthetic and observed features, exact coincidence is not expected due to the spectral averaging introduced by the low-resolution sampling designed into the TES instrument. The peaks around 400-500 $\mathrm{cm}^{-1}$ were 

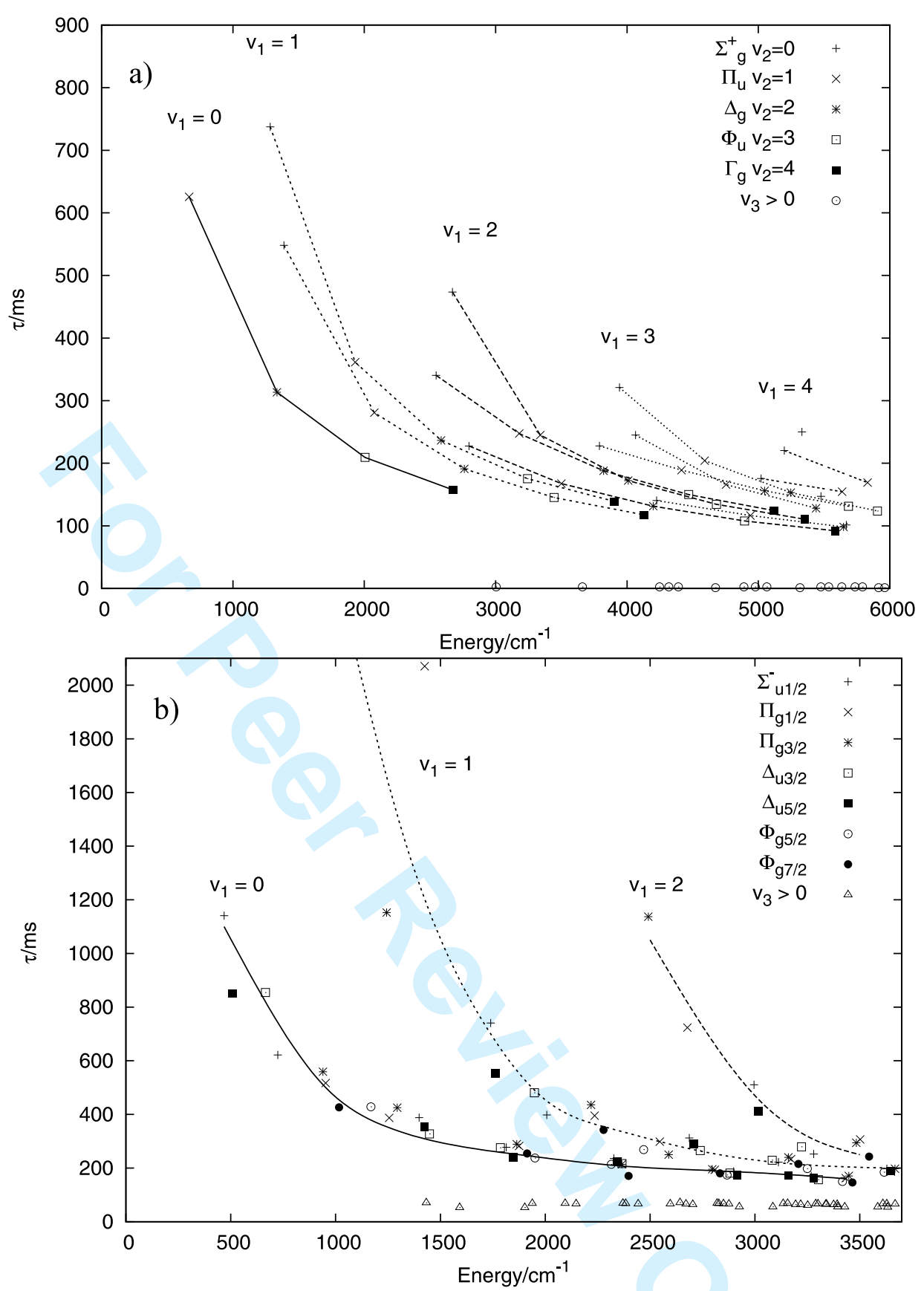

FIG. 8: a) $\mathrm{CO}_{2}$ rovibrational radiative lifetimes for levels with $J=|P|=l$ and $0 \leq v_{2} \leq 4$. The points associated with the same value of $v_{1}$ and the same position in the polyads are linked. The radiative lifetime $\tau_{n}$ of the level $n$ is given by $\tau_{n}=\left(\sum_{n^{\prime}} A_{n n^{\prime}}\right)^{-1}$ where $A_{n n^{\prime}}$ is the Einstein coefficient for spontaneous emission and $E_{n^{\prime}}<E_{n}$. b) CO $C_{2}^{+}$rovibrational radiative lifetimes for levels with $J=|P|$ and $0 \leq v_{2} \leq 6$. Averaged curves, passing through the points associated with the same value of $v_{1}$ are shown.

assigned to the emission of the $\mathrm{CO}_{2}^{+}$bending levels. The remaining unassigned peaks are expected to be due to emission of bending levels of other neutral or ionic polyatomic species.

\section{CONCLUSIONS}

The theoretical radiative transition probabilities within the electronic states of molecular ions can be calculated with sufficient precision for most applications in molecular physics. Progress may be expected in the development of new experimental techniques for more reliable electric dipole moment functions of molecular ions. 


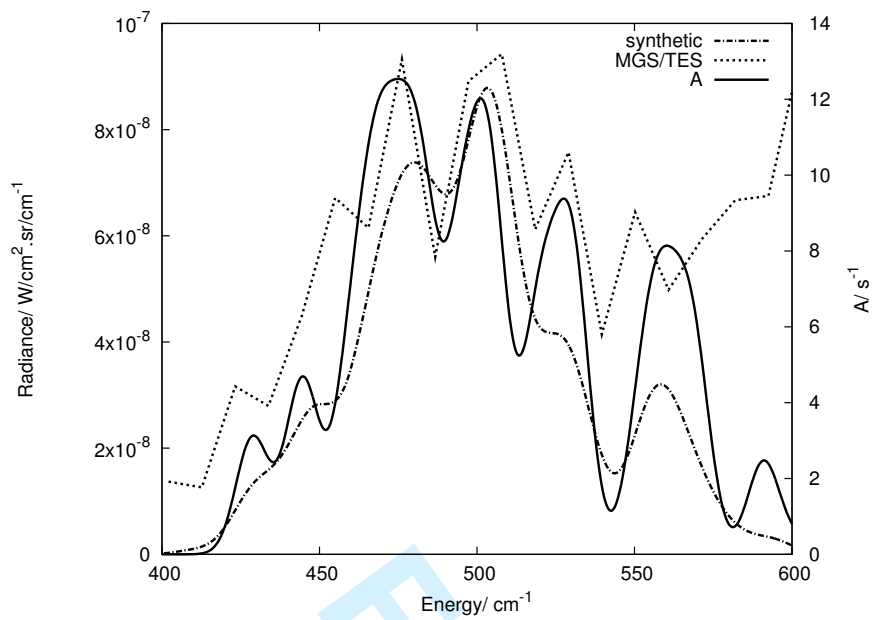

FIG. 9: Mars Global Surveyor thermal emission spectra. The dotted line curve is a night spectrum with random error about $10^{-9} \mathrm{~W} / \mathrm{cm}^{2} \cdot \mathrm{sr} / \mathrm{cm}^{-1}$. It is an average over $30^{\circ} \mathrm{S}$ to $30^{\circ} \mathrm{N}$ latitude, $42-84 \mathrm{~km}$. The synthetic spectrum (broken curve) is computed for a tangent altitude of $50 \mathrm{~km}$ and assumes Boltzmann populations. The solid line curve represents the ab initio spectrum obtained with the spontaneous emission rates (cf. text and Ref. [3]).
In this challenging task, the interplay between theory and experiment will remain essential.
[1] M. Diehr, P. Rosmus, S. Carter and P.J. Knowles Mol. Phys., 102, 2181 (2004) and references therein.

[2] T. Cours, P. Rosmus and Vl. G. Tyuterev J. Chem. Phys., 117, 5192 (2002) and references therein.

[3] C. Léonard, M. Diehr, P. Rosmus and W.C. Maguire Journal of Quantitative Spectroscopy nad Radiative transfer, 109535 (2007) and references therein.

[4] C. P. Rinsland, J. -M. Flaud, A. Perrin, M. Birk, G. Wagner, A. Goldman, A. Barbe, M. - R. De BackerBarilly, S. N. Mikhailenko, Vl. G. Tyuterev, M. A. H. Smith, V. Malathy Devi, D. Chrisz Benner, F. Schreier, K. V. Chance, J. Orphal, T. M. Stephen, 2003, J. Quant. Spectrosc. and Radiat. Transfer, 82, 207, and references therein.

[5] L. S. Rothman, A. Barbe, D. C. Benner, L. R. Brown, C. Camy-Peyret, M. R. Carleer, K. Chance, C. Clerbaux, V. Dana, V. M. Devi, A. Fayt, J. - M. Flaud, R. R. Gamache, A. Goldman, D. Jacquemart, K. W. Jucks, W. J. Lafferty, J. Y. Mandin, S. T. Massie, V. Nemtchinov, D. A. Newnham, A. Perrin, C. P. Rinsland, J. Schroeder, K. M. Smith, M. A. H. Smith, K. Tang, R. A. Toth, J. Vander Auwera, P. Varanasi, and K. Yoshino, J. Quant. Spectrosc. Radiat. Transfer, 82, 5 (2003) and references therein.

[6] S. Mikhailenko, Yu. Babikov, Vl. G. Tyuterev, A. Barbe, The Database of Ozone Spectroscopy on WEB (S\&MPO), Computational Technologies, 2002, v.7, pp.64-70; S\&MPO Information system v1.1 available at http://ozone.iao.ru, and references therein.

[7] All electronic structure calculations were performed with MOLPRO package of ab initio programs of H.-J. Werner, and P.-J. Knowles. Further information can be obtained from http://www.molpro.net/
[8] S. Carter, N.C. Handy, and B.T. Sutcliffe, Mol. Phys., 49, 745 (1983).

[9] S. Carter, J. Senekowitsch, N. C. Handy, and P. Rosmus, Mol. Phys., 65, 143 (1988).

[10] W. Gabriel, E. - A. Reinsch, P. Rosmus, S. Carter, and N. C. Handy, J. Chem. Phys., 99, 897 (1993).

[11] J. - M. Flaud, and R. Bacis, Spectrochimica Acta, A 54, 3 (1998) and references therein.

[12] O. N. Sulakshina, Yu. G. Borkov, and Vl. G.Tyuterev, Vl. G J. Chem. Phys. 113, 10572 (2000) and references therein.

[13] D. Xie, H. Guo, and K. A. Peterson, J. Chem. Phys. 112, 8378 (2000) and references therein.

[14] J. K. Thompson, S. Rainville, and D. E. Pritchard, Nature (london), 430, 58 (2004).

[15] M. Cheng, J.M. Brown, P. Rosmus, R. Linguerri, N. Komiha and E.G. Myers , Phys. Rev. A,75, 012502 (2007) and references therein.

[16] K. B. Laughlin, G. A. Blake, R. C. Cohen, D. C. Hovde, and R. J. Saykally, Phys. Rev. Lett. 58, 996 (1987).

[17] K. B. Laughlin, G. A. Blake, R. C. Cohen, and R. J. Saykally, J. Chem. Phys. 90, 1358 (1989).

[18] M. Molski, Chem. Phys. Lett. 342, 293 (2001).

[19] E. A. Engel, N. Doss, G. J. Harris, and J. Tennyson, Mon. Not. R. Astron. Soc. 357, 471 (2005),

[20] T. Wyttenbach, C. G. Beggs, and M. T. Bowers, Chem. Phys. Lett. 177, 239 (1991).

[21] G. Chambaud, and P. Rosmus, Chem. Phys. Lett. 165, 429 (1990).

[22] H. J. Wörner, R. van der Veen, and F. Merkt, Phys. Rev. Lett. 97, 173003 (2006). 


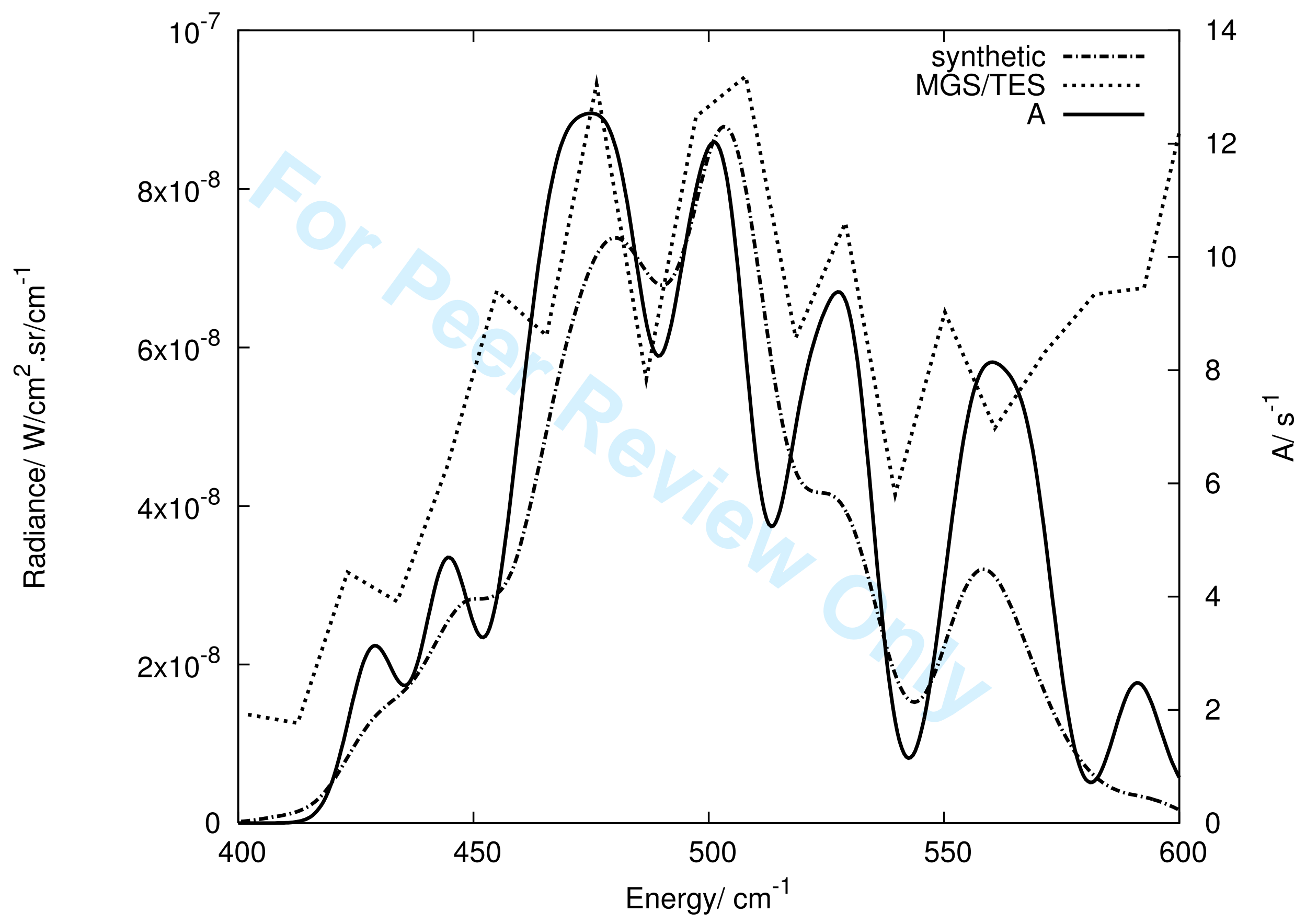

URL: http://mc.manuscriptcentral.com/tandf/tmph 


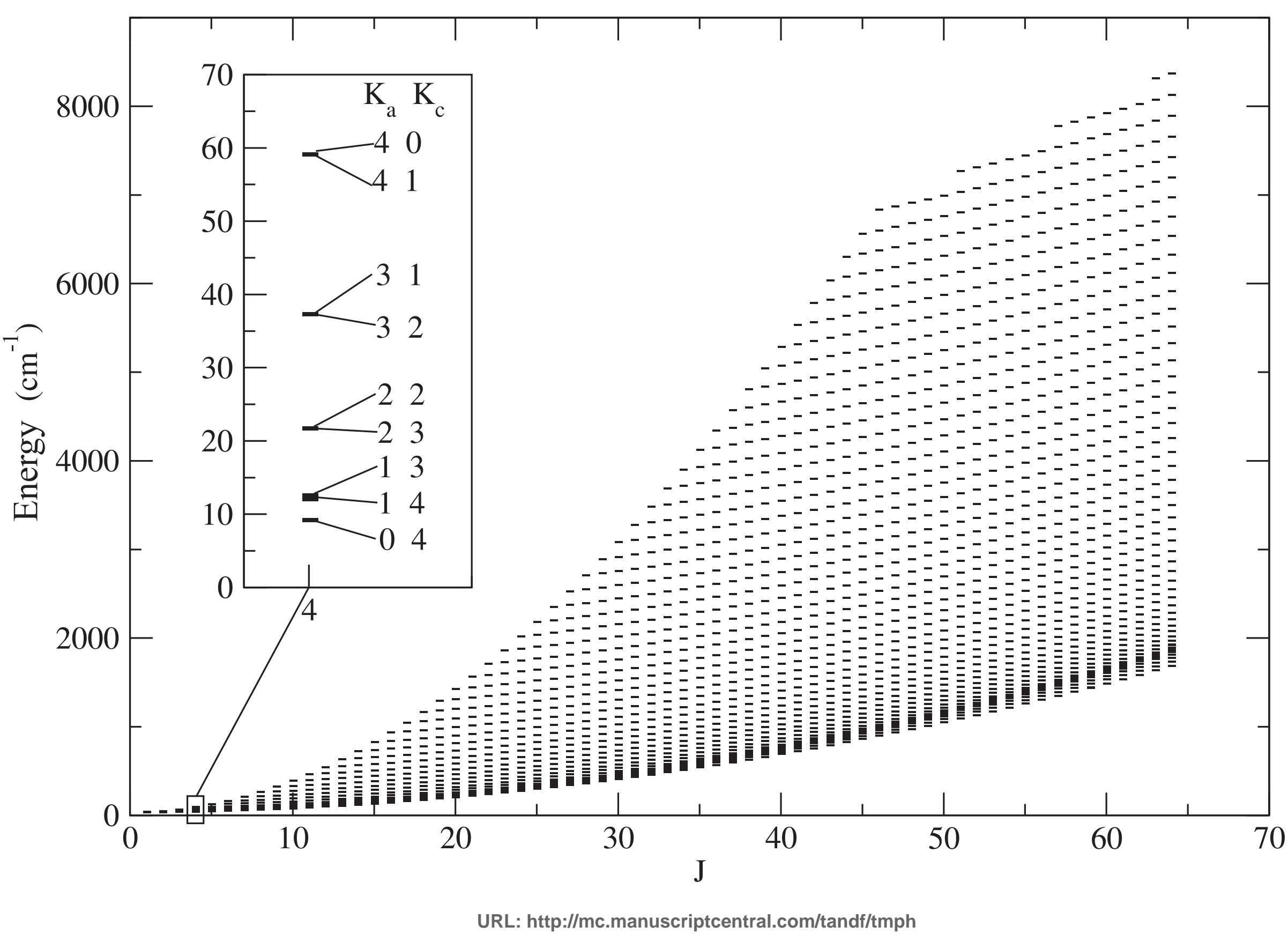




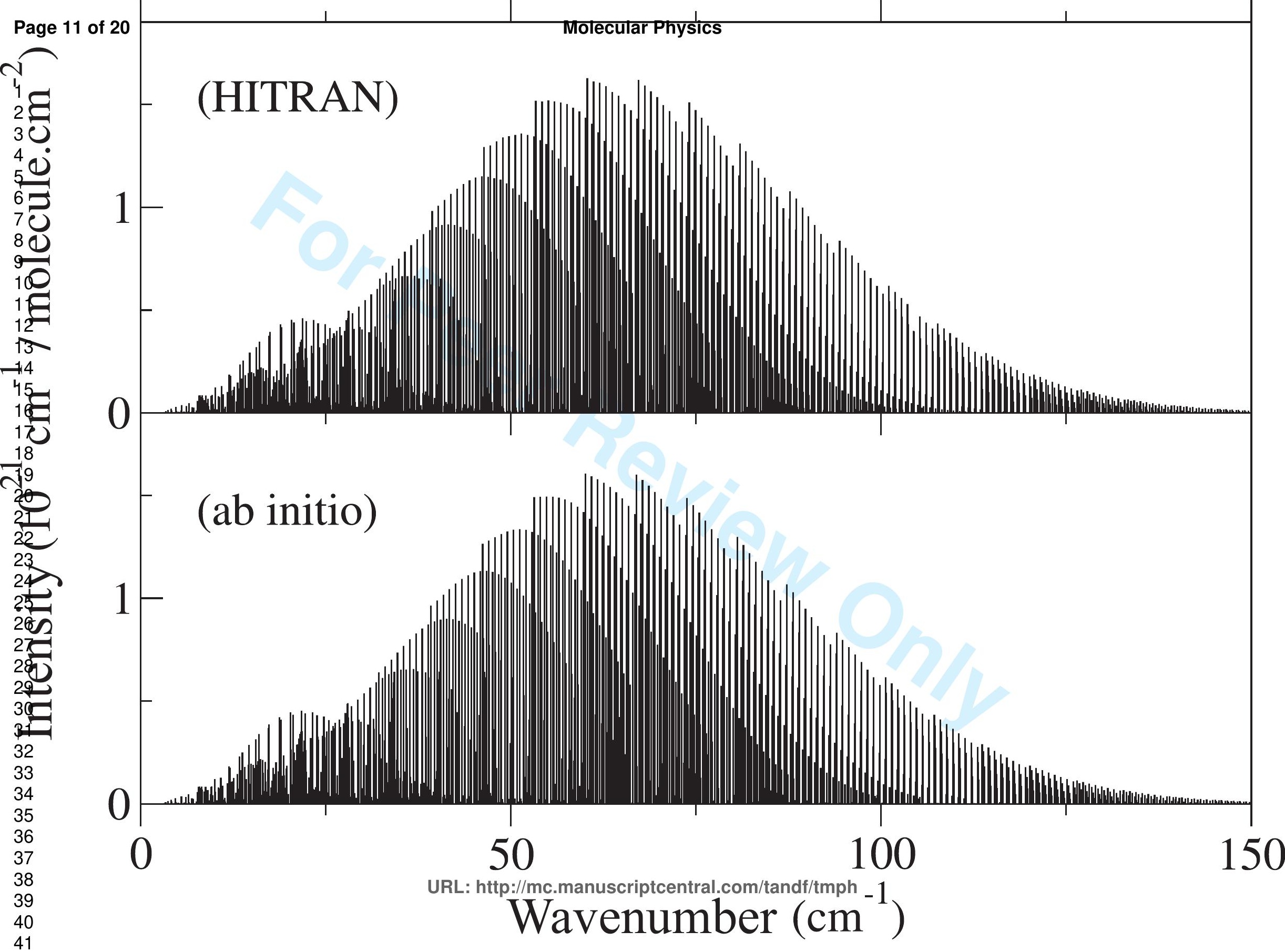




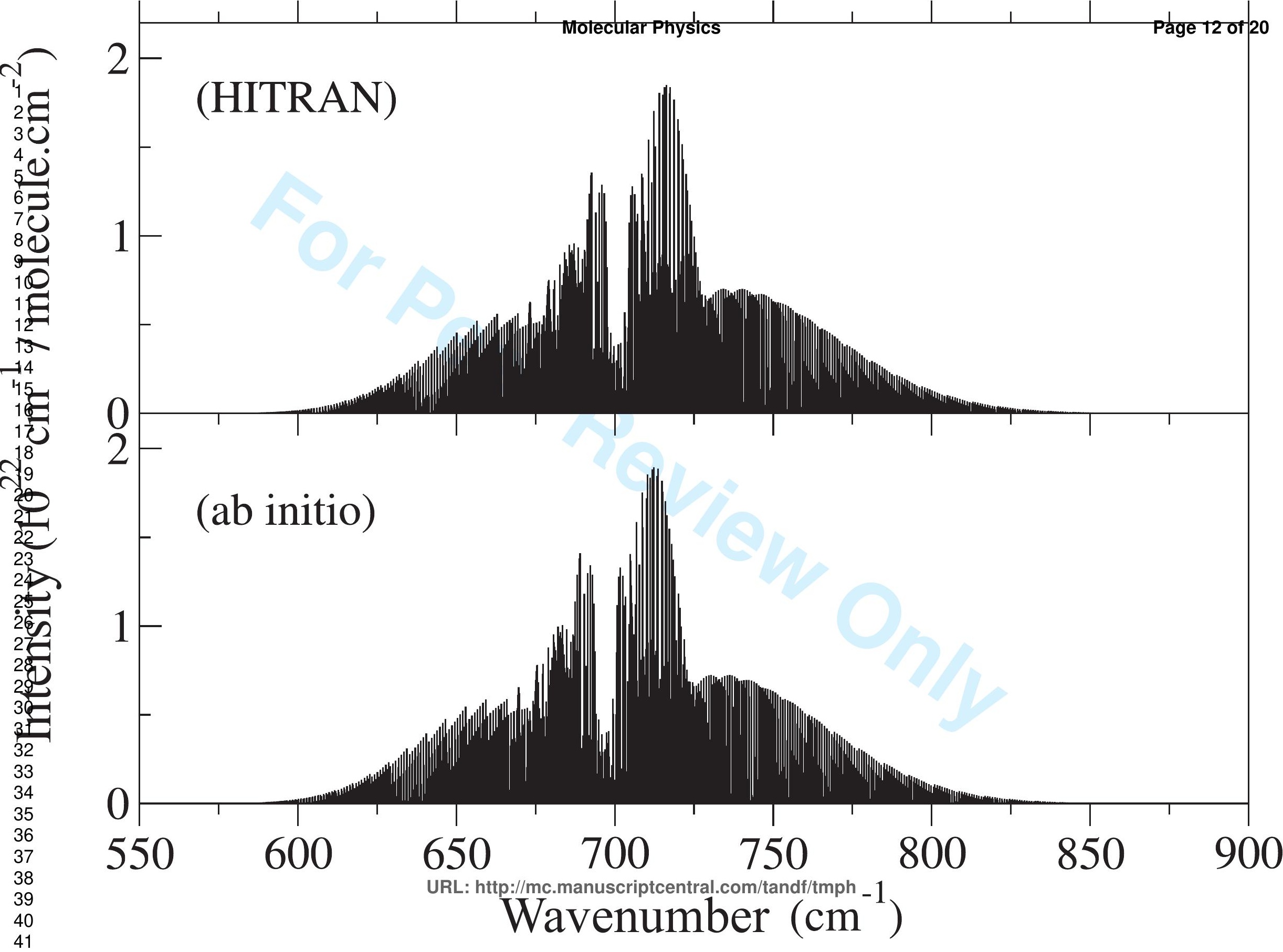




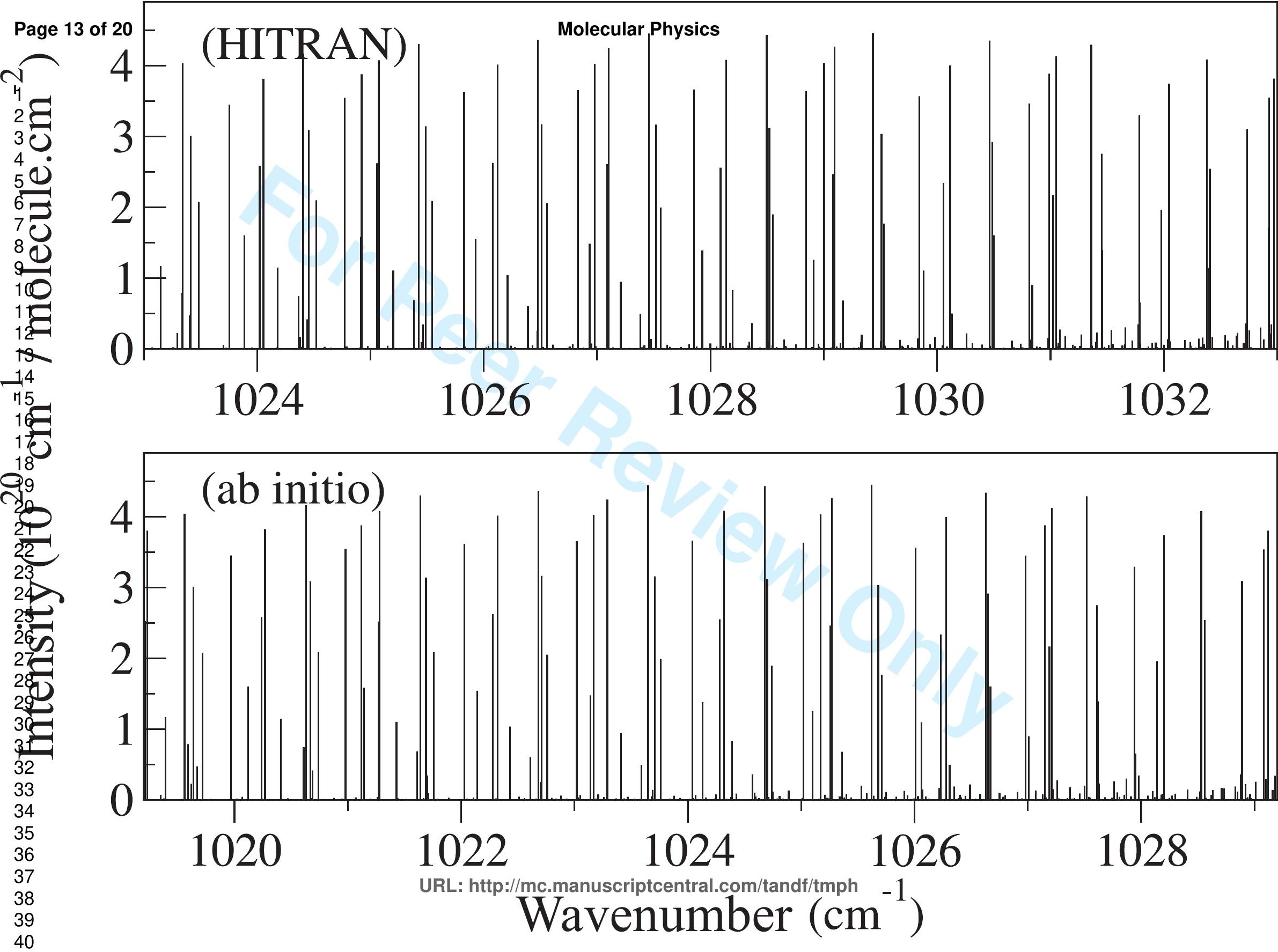




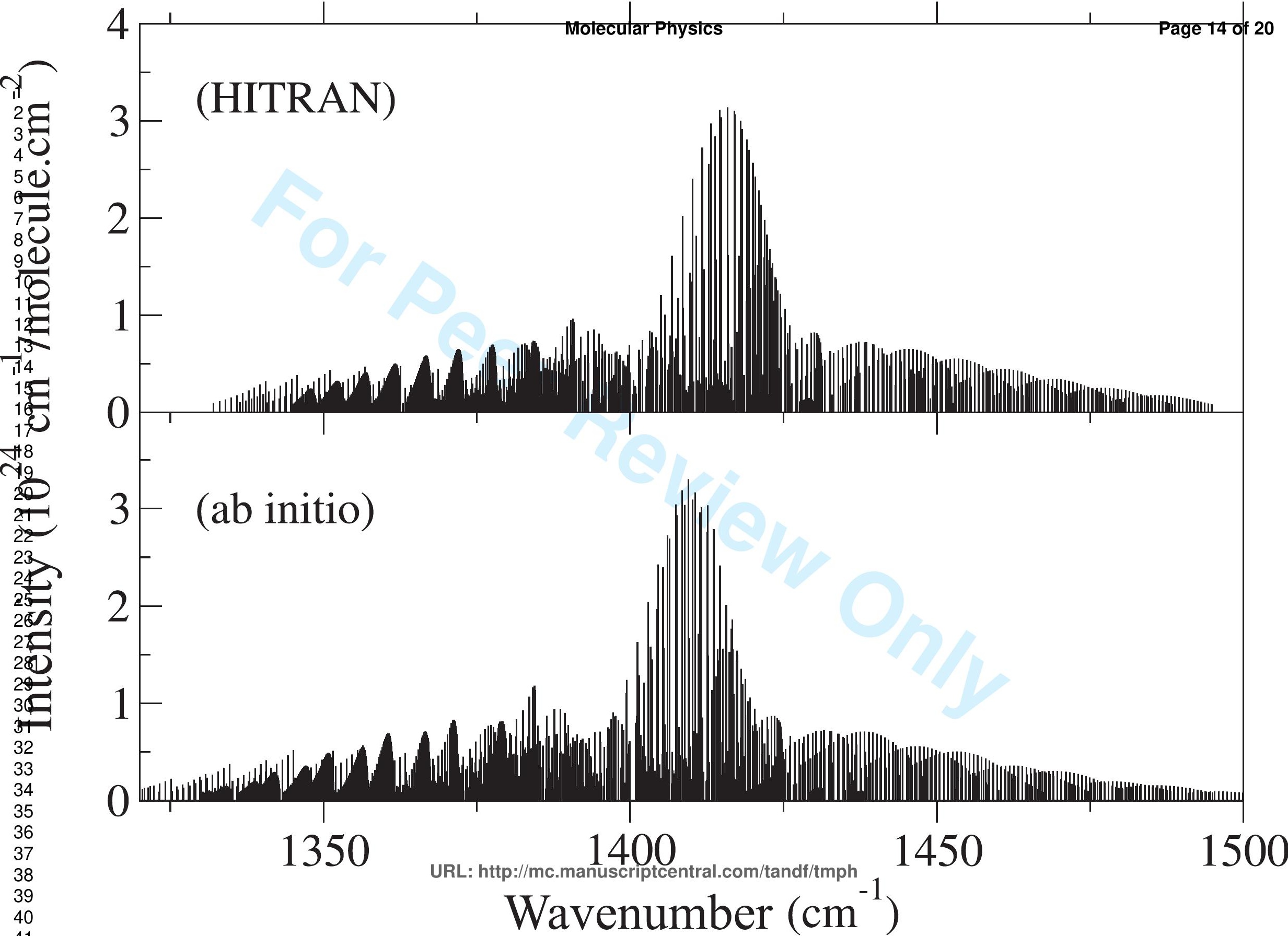




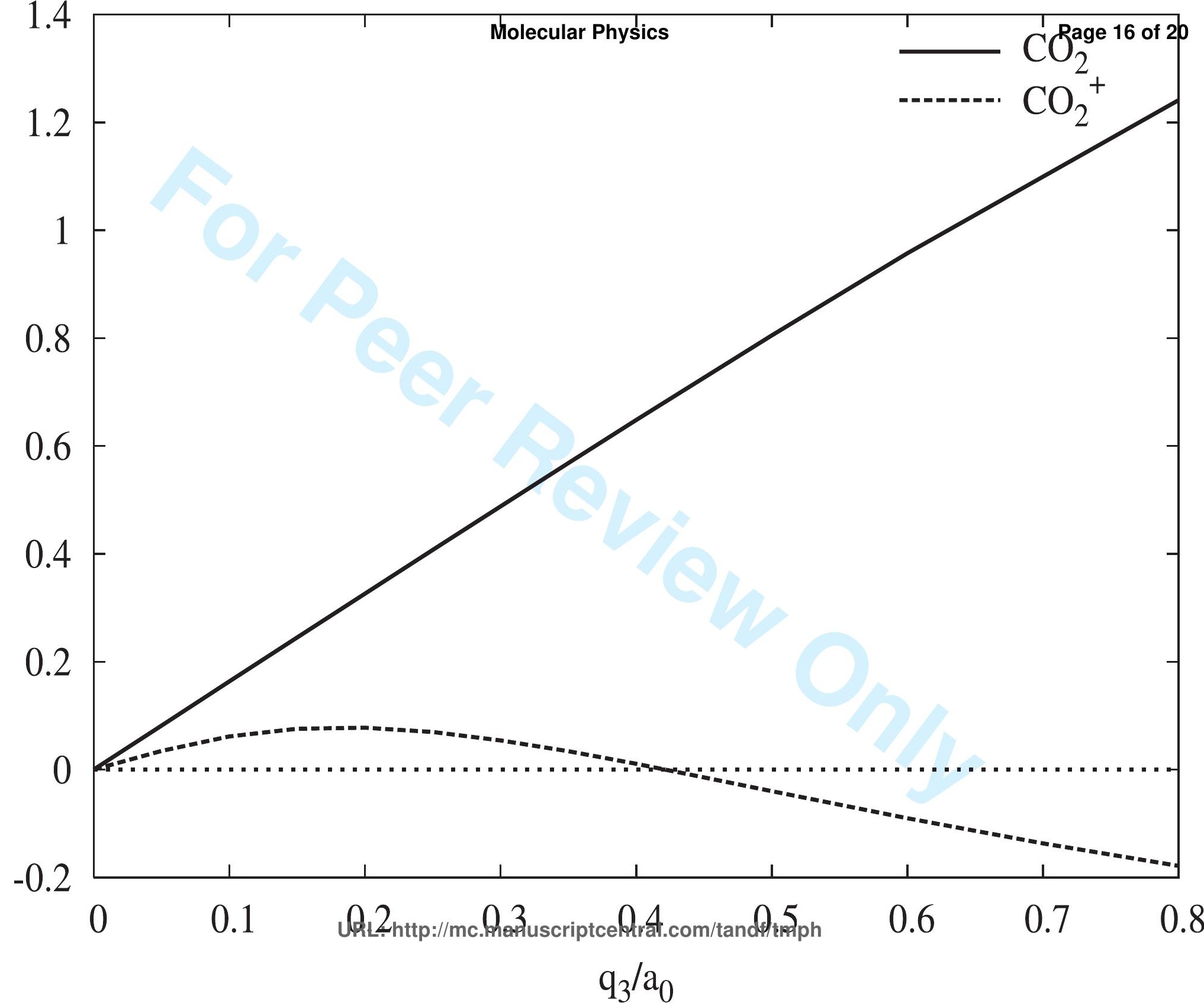




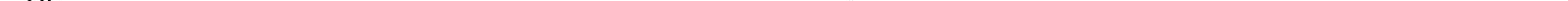




\section{Page 19 of 20}


\section{Molecular Physics}

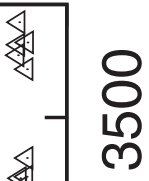

2

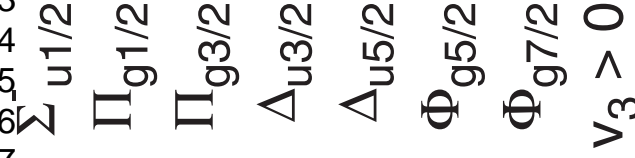

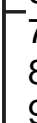

10

12

$-13$

15

16

18

19

21

2

2

25

2

2

30

31

$-3$

34

35

37

38

41

42

43

44

45

4

4

$5 C$

51

5

54 


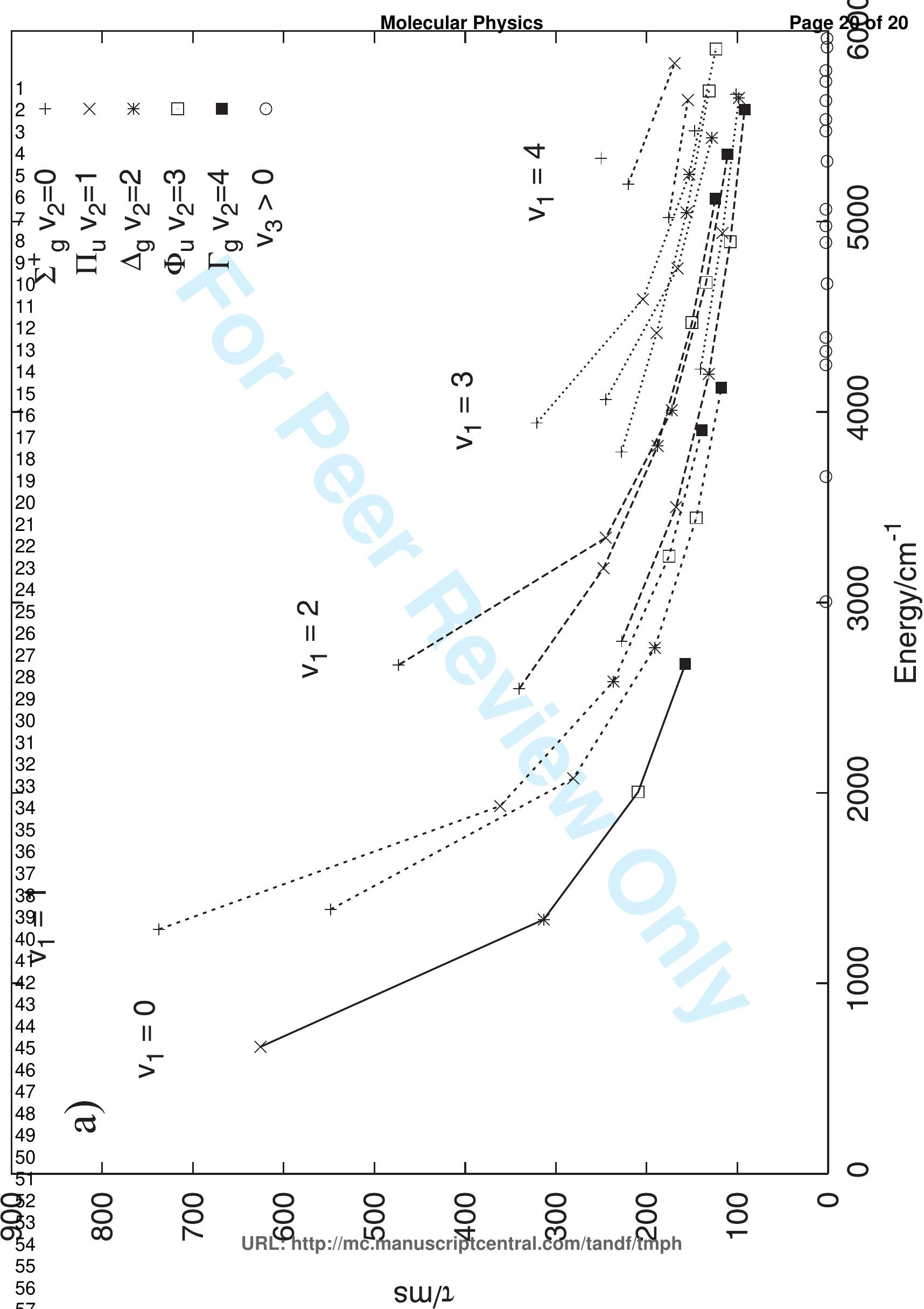

